\title{
Continuous-Time Mean-Variance Portfolio Selection with Partial Information
}

\author{
Wan-Kai Pang1, Yuan-Hua Ni², Xun Li' ${ }^{1}$, Ka-Fai Cedric Yiu ${ }^{1}$ \\ ${ }^{1}$ Department of Applied Mathematics, The Hong Kong Polytechnic University, Hong Kong, China \\ ${ }^{2}$ School of Sciences, Tianjin Polytechnic University, Tianjin, China \\ Email: macyiu@polyu.edu.hk
}

Received 3 August 2014; revised 1 October 2014; accepted 21 October 2014

Copyright (C) 2014 by authors and Scientific Research Publishing Inc.

This work is licensed under the Creative Commons Attribution International License (CC BY).

http://creativecommons.org/licenses/by/4.0/

(c) (i) Open Access

\begin{abstract}
This paper studies a continuous-time market under a stochastic environment where an agent, having specified an investment horizon and a target terminal mean return, seeks to minimize the variance of the return with multiple stocks and a bond. In the model considered here, the mean returns of individual assets are explicitly affected by underlying Gaussian economic factors. Using past and present information of the asset prices, a partial-information stochastic optimal control problem with random coefficients is formulated. Here, the partial information is due to the fact that the economic factors can not be directly observed. Using dynamic programming theory, we show that the optimal portfolio strategy can be constructed by solving a deterministic forward Riccati-type ordinary differential equation and two linear deterministic backward ordinary differential equations.
\end{abstract}

\section{Keywords}

Mean-Variance Portfolio Selection, Partial Information, Filtering

\section{Introduction}

Mean-variance is an important investment decision rule in financial portfolio selection, which is first proposed and solved in the single-period setting by Markowitz in his Nobel-Prize-winning works [1] [2]. In these seminal papers, the variance of the final wealth is used as a measure of the risk associated with the portfolio and the agent seeks to minimize the risk of his investment subject to a given mean return. This model becomes the foundation of modern finance theory and inspires hundreds of extension and applications. For example, this leads to the elegant capital asset pricing model [3].

The dynamic extension of the Markowitz model has been established in subsequent years by employing the 
martingale theory, convex duality and stochastic control. The pioneer work for continuous time portfolio management is [4], in which Merton used dynamic programming and partial differential equation (PDE) theory to derive and solve the Hamilton-Jacobi-Bellman (HJB) equation, and thus obtains the optimal strategy. For cases when the underlying stochastic process is a Martingale, optimal portfolios could be derived [5]. In [6], the authors formulated the mean-variance problem with deterministic coefficients as a linear-quadratic (LQ) optimal problem. As there is no running cost in the objective function, this formulation is inherently an indefinite stochastic LQ control problem. As extensions of [6], for example, [7] dealt with random coefficients, while [8] considered regime switching market. For discrete time cases, [9] solved the multiperiod mean-variance portfolio selection problem completely. Analytical optimal strategy and an efficient algorithm to find the strategy were proposed. Comprehensive review of the mean-variance model can be found in [10] and [11].

In [12], in order to tackle the computational tractability and the statistical difficulties associated with the estimation of model parameters, Bielecki and Pliska introduced a model such that the underlying economic factors such as accounting ratios, dividend yields, and macroeconomic measures are explicitly incorporated in the model. The factors are assumed to follow Gaussian processes and the drifts of the stocks are linear functions of these factors. This model motivates many further researches (see, for example, [13] and [14]). In practice, many investors use only the observed asset prices to decide his current portfolio strategy. The random factors cannot normally be observable directly. Therefore, the underlying problem falls into the category of portfolio selection under partial information [15] [16]. A significant progress in the realm of mean-variance concerning partial information is the work of [17], in which a separation principle is shown under this partial information setting. Efficient strategies were derived, which involved the optimal filter of the stock drift processes. In addition, the particle system representation of the obtained filter is employed to develop analytical and numerical approaches. It is valuable to point out that backward stochastic differential equations (BSDEs) methodology is employed to tackle this problem.

This paper attempts to deal with the mean-variance portfolio selection under partial information based on the model of [12]. By exploiting the properties of the filtering process and the wealth process, we tackle this problem directly by the dynamic programming approach. We show that optimal strategy can be constructed by solving a deterministic forward Riccati-type ordinary differential equation (ODE) and a system of linear deterministic backward ODEs. Clearly, by reversing the time, a deterministic backward ODE can be converted to a forward one. Therefore, we can easily derive the analytic solutions of the ODEs, and thus the analytic form of the optimal strategies. This is the main contribution of the paper. The proposed procedure is different from that of [17], where BSDEs are employed.

The rest of the paper is organized as follows. In Section 2, we formulate the mean-variance portfolio selection model under partial information, and an auxiliary problem is introduced. Section 3 gives the optimal strategy of the auxiliary problem by the dynamic programming method. Section 4 studies the original problem, while Section 5 gives some concluding remarks.

\section{Mean-Variance Model}

Throughout this paper $\left(\Omega, \mathcal{F}, P,\left\{\mathcal{F}_{t}\right\}_{t \geq 0}\right)$ is a fixed filtered complete probability space on which a standard $\mathcal{F}_{t}$ adapted $(n+m)$-dimensional Brownian motion $\{W(t), t \geq 0\}$ is defined, where $W(t) \equiv\left(W^{1}(t), \cdots, W^{n+m}(t)\right)^{\mathrm{T}}$ and $W(0)=0$. Let $T>0$ be the terminal time of an investment, and $L_{\mathcal{F}}^{2}\left(0, T ; \mathbb{R}^{d}\right)$ denotes the set of all $\mathbb{R}^{d}$-valued, $\mathcal{F}_{t}$-adapted stochastic processes $f(t)$ with $E \int_{0}^{T}|f(t)|^{2} \mathrm{~d} t<+\infty$; similarly $L_{\mathcal{H}}^{2}\left(0, T ; \mathbb{R}^{l}\right)$ can be defined for any functions with domain in $\mathbb{R}^{l}$ and filtration $\mathcal{H}_{t}$.

There is a capital market containing $m+1$ basic securities (or assets) and $n$ economic factors. The securities consist of a bond and $m$ stocks. The set of factors may include short-term interests, the rate of inflation, and other economic factors [14]. One of the securities is a risk-free bank account whose value process $S_{0}(t)$ is subject to the following ordinary differential equation

$$
\mathrm{d} S_{0}(t)=r(t) S_{0}(t) \mathrm{d} t, \quad t \geq 0, \quad S_{0}(0)=s_{0}>0
$$


where $r(t)$ is the interest rate, a deterministic function of $t$. The other $m$ assets are risky stocks whose price processes $S_{1}(t), \cdots, S_{m}(t)$ satisfy the following stochastic differential equations (SDEs)

$$
\begin{cases}\mathrm{d} S_{i}(t)=S_{i}(t)\left\{\mu_{i}(t) \mathrm{d} t+\sum_{j=1}^{n+m} \sigma_{i j}(t) \mathrm{d} W^{j}(t)\right\}, & t \geq 0, \\ S_{i}(0)=S_{i}>0, & i=1,2, \cdots, m\end{cases}
$$

where $\mu_{i}(t), i=1, \cdots, m$ are the drifts, and $\sigma_{i}(t), i=1, \cdots, m$ are the deterministic volatility or dispersion rate of the stocks. In this paper, we assume that the drifts are affine functions of the mentioned economic factors, and the factors are Gaussian processes. To be precise, denoting $\left(\mu_{1}(t), \mu_{2}(t), \cdots, \mu_{m}(t)\right)^{\mathrm{T}}$ by $\mu(t)$, we have

$$
\left\{\begin{array}{l}
\mu(t)=a+A y(t) \triangleq \mu^{y}(t), \quad a \in \mathbb{R}^{m}, \quad A \in \mathbb{R}^{m \times n}, \\
\mathrm{~d} y(t)=(\bar{d}+D y(t)) \mathrm{d} t+\Lambda \mathrm{d} W(t), \quad y(0)=y_{0} \in \mathbb{R}^{n}
\end{array}\right.
$$

where the constant matrices $\bar{d}, D, \Lambda$ are of $n \times 1, n \times n, n \times(m+n)$, respectively.

Consider an agent with an initial endowment $x_{0}>0$ and an investment horizon $[0, T]$, whose total wealth at time $t \in[0, T]$ is denoted by $X(t)$. Assuming that the trading of shares is self-financed and taken place continuously, and that transaction cost and consumptions are not considered, then $X(t)$ satisfies (see, e.g., [18])

$$
\left\{\begin{array}{l}
\mathrm{d} X(t)=\left\{r(t) X(t)+\sum_{i=1}^{m}\left[\mu_{i}^{y}(t)-r(t)\right] \pi_{i}(t)\right\} \mathrm{d} t+\sum_{i=1}^{m} \sum_{j=1}^{n+m} \pi_{i}(t) \sigma_{i j}(t) \mathrm{d} W^{j}(t), \\
X(0)=x_{0}
\end{array}\right.
$$

where $\pi_{i}(t), \quad i=1, \cdots, m$ denote the total market value of the agent's wealth in the $i$-th stock. We call the process $\pi(t)=\left(\pi_{1}(t), \cdots, \pi_{m}(t)\right)^{\mathrm{T}}, 0 \leq t \leq T$, a portfolio of the agent.

Let

$$
\begin{aligned}
& S(t)=\left(S_{1}(t), \cdots, S_{m}(t)\right)^{\mathrm{T}}, \\
& \mathcal{G}_{t}=\sigma\left(S_{0}(u), S(u): u \leq t\right), t \geq 0 .
\end{aligned}
$$

As pointed out by [17], practically, the investor can only observe the prices of assets. So, at time $t$, the information that available to the investor is the past and present assets' prices, equivalently, the filtration $\mathcal{G}_{t}$. Thus, the investor's strategy should be based on his/her available information. Therefore, $\pi_{t}$ should be $\mathcal{G}_{t}$ measurable. To be exact, we define the following admissible portfolio.

Definition 2.1. A portfolio $\pi(\cdot)$ is said to be admissible if $\pi(\cdot) \in L_{\mathcal{G}}^{2}\left(0, T, \operatorname{Re}^{m}\right)$ and the SDE (2.3) has a unique solution $x(\cdot)$ corresponding to $\pi(\cdot)$. The totality of all admissible portfolios is denoted by $\Pi$.

The agent's objective is to find an admissible portfolio $\pi(\cdot)$, among all such admissible portfolios that his/her expected terminal wealth $E X(T)=\bar{x}$, where $\bar{x} \geq x_{0} \mathrm{e}^{\int_{0}^{T} r(t) \mathrm{d} t}$ is given a priori, so that the risk measured by the variance of the terminal wealth

$$
\operatorname{Var} X(T):=E[X(T)-E X(T)]^{2} \equiv E[X(T)-\bar{x}]^{2}
$$

is minimized. The problem of finding such a portfolio $\pi(\cdot)$ is referred to as the mean-variance portfolio selection problem. Mathematically, we have the following formulation.

Definition 2.2. The mean-variance portfolio selection problem, with respect to the initial wealth $x_{0}$, is formulated as a constrained stochastic optimization problem parameterized by $\bar{x} \geq x_{0} \mathrm{e}^{\int_{0}^{T} r(t) d t}$ : 


$$
\begin{cases}\text { minimize } & \operatorname{Var} X(T)=E[X(T)-\bar{x}]^{2}=E\left[X(T)^{2}\right]-\bar{x}^{2}, \\ \text { subject to } & \begin{cases}X(0)=x_{0}, & E X(T)=\bar{x} \\ (X(\cdot), \pi(\cdot)) & \text { admissible. }\end{cases} \end{cases}
$$

The problem is called feasible (with respect to $\bar{x}$ ) if there is at least one admissible portfolio satisfying $E X(T)=\bar{x}$. An optimal portfolio, if it exists, is called an efficient portfolio strategy with respect to $\bar{x}$, and $\operatorname{Var} X(T)$ is called an efficient point. The set of all efficient points is obtained when the parameter $\bar{X}$ varies between $\left[x_{0} \mathrm{e}^{\int_{0}^{T} r(t) \mathrm{d} t},+\infty\right)$.

We impose the basic assumption:

Assumption (PD). For any $t \geq 0, \sigma(t) \sigma^{\mathrm{T}}(t)>0$, which is popular in the literatures about portfolio selection (see, for example, [12]-[14] [17] [19]).

Let

$$
B(t) \triangleq\left(\mu^{y}(t)\right)^{\mathrm{T}}-r(t) \mathbf{1}, \quad \sigma(t)=\left(\sigma_{i j}(t)\right)_{m \times(n+m)},
$$

with 1 being a $m$-dimensional row vector with all its entries being 1. Then, (2.3) can be rewritten as

$$
\left\{\begin{array}{l}
\mathrm{d} X(t)=[r(t) X(t)+B(t) \pi(t)] \mathrm{d} t+\pi(t)^{\prime} \sigma(t) \mathrm{d} W(t), \\
X(0)=x_{0} .
\end{array}\right.
$$

By the definition of $\pi$, our problem falls into the category of stochastic control based on partial information. Here, the partial information means that we cannot know the process $y(t)$, and thus $B(t)$. In order to design admissible strategy, we firstly need to derive the optimal estimation of $y_{t}$. Let

$$
\begin{aligned}
& \Gamma=\sigma(t) \sigma^{\mathrm{T}}(t) \in \mathbb{R}^{m \times m}, \\
& \bar{\Gamma}=\left(\Gamma_{11}(t), \cdots, \Gamma_{n n}(t)\right)^{\mathrm{T}}, \quad \Sigma(t)=\Gamma^{\frac{1}{2}}(t)=\Sigma^{\mathrm{T}}(t), \\
& Y(t)=\left(\log S_{1}(t), \cdots, \log S_{m}(t)\right)^{\mathrm{T}} \triangleq \log S(t) .
\end{aligned}
$$

By Itô's formula we have

$$
\mathrm{d} Y(t)=\left(a+A y(t)-\frac{1}{2} \bar{\Gamma}(t)\right) \mathrm{d} t+\sigma(t) \mathrm{d} W_{t}, \quad Y_{0}=\log S(0) .
$$

Define

$$
\mathrm{d} v(t)=\Sigma^{-1}\left[\mathrm{~d} Y(t)-\left(a+A \hat{y}(t)-\frac{1}{2} \bar{\Gamma}(t)\right) \mathrm{d} t\right]
$$

then $\{v(t), t \geq 0\}$ is a Brownian motion under the original probability measure (Liptser and Shiryaev (2001)). The estimation of $y(t)$ is given by (Theorem 10.3 of [20])

$$
\left\{\begin{array}{l}
\mathrm{d} \hat{y}(t)=(\bar{d}+D \hat{y}(t)) \mathrm{d} t+\left(\Lambda \sigma^{\mathrm{T}}+\beta(t) A^{\mathrm{T}}\right)\left(\Sigma^{\mathrm{T}}\right)^{-1} \mathrm{~d} v(t), \\
\dot{\beta}(t)=D \beta(t)+\beta(t) D^{\mathrm{T}}+\Lambda \Lambda^{\mathrm{T}}-\left(\Lambda \sigma^{\mathrm{T}}+\beta(t) A^{\mathrm{T}}\right)\left(\Sigma \Sigma^{\mathrm{T}}\right)^{-1}\left(\Lambda \sigma^{\mathrm{T}}+\beta(t) A^{\mathrm{T}}\right)^{\mathrm{T}}, \\
\hat{y}(0)=y_{0}, \quad \beta(0)=0 .
\end{array}\right.
$$

By (2.7), a simple calculation shows that

$$
\sigma(t) \mathrm{d} W(t)=\Sigma(t) \mathrm{d} v(t)+A[\hat{y}(t)-y(t)] .
$$

Substituting (2.9), we have an equivalent representation of the wealth process 


$$
\left\{\begin{array}{l}
\mathrm{d} X(t)=[r(t) X(t)+\bar{B}(t) \pi(t)] \mathrm{d} t+\pi(t)^{\prime} \Sigma(t) \mathrm{d} v(t), \\
X(0)=x_{0}
\end{array}\right.
$$

where

$$
\bar{B}(t)=(a+A \hat{y}(t))^{\mathrm{T}}-r(t) \mathbf{1}
$$

This is the separation principle developed by [17], which enables us to solve problem (2.5) as if the drifts $\mu^{y}(\cdot)$ were known, and then replace $\mu^{y}(\cdot)$ by its optimal estimation. So, (2.5) can be equivalently formulated as

$$
\begin{cases}\text { minimize } & E\left[(X(T)-\bar{x})^{2}\right] \\
\text { subject to } & \left\{\begin{array}{l}
E X(T)=\bar{X} \\
\pi \in \Pi, \\
(X(\cdot), \pi(\cdot)) \text { satisfy }(2.8)(2.10)(2.11) .
\end{array}\right.\end{cases}
$$

By general convex optimization theory, the constrained optimal problem (12) with $(E X(T)=\bar{x})$ can be converted into an unconstrained one by introducing a Lagrange multiplier $\gamma$. To be concrete, for any fixed $\gamma$, we consider the following problem

$$
\begin{cases}\text { minimize } & E[X(T)-\bar{X}]^{2}-2 \gamma E[X(T)-\bar{x}]=E[X(T)-\gamma-\bar{x}]^{2}-\gamma^{2}, \\
\text { subject to } & \left\{\begin{array}{l}
\pi \in \Pi, \\
(X(\cdot), \pi(\cdot)) \text { satisfy }(2.8)(2.10)(2.11)
\end{array}\right.\end{cases}
$$

which is equivalent to the following (denoting $\bar{x}+\gamma$ by $\alpha$ for any fixed $\gamma$ )

$$
\begin{cases}\text { minimize } & \frac{1}{2} E\left[(X(T)-\alpha)^{2}\right] \\
\text { subject to } & \left\{\begin{array}{l}
\pi \in \Pi, \\
(X(\cdot), \pi(\cdot)) \text { satisfy }(2.8)(2.10)(2.11)
\end{array}\right.\end{cases}
$$

in the sense that two problems have exactly the same optimal strategy. In the following, we will call problem (2.14) the auxiliary problem of the original problem (2.12).

\section{Optimal Policy for the Auxiliary Problem}

The problem (2.14) can be viewed as an unconstrained special stochastic optimal control problem with random coefficients in system equation and zero integral term in the performance index. Different from existing results using BSDEs methodology, in this section, we derive the optimal portfolio strategy from dynamic programming directly. This enables us to derive the optimal policy by solving just two linear deterministic backward ODEs and a Riccati-type forward deterministic ODE.

\subsection{Analysis of Hamilton-Jacobi-Bellman Equation}

Let $J(t, X, \hat{y})$ denote the performance of problem (2.14) at time $t$, with boundary condition $J(T, X, \hat{y})=\frac{1}{2} E\left[(X(T)-\alpha)^{2}\right]$. Then, it is evident that the following HJB equation is satisfied

$$
\min _{\pi \in \Pi}(\mathcal{L} J)(t, X, \hat{y})=0, \quad J(T, X, \hat{y})=\frac{1}{2}(X-\alpha)^{2}
$$

where $\mathcal{L}$ is the infinitesimal generator operator of the closed system (2.8) (2.10) (2.11), and the independence of $X$ on policy $\pi$ is suppressed.

To evaluate $\mathcal{L}$, first of all, by (2.8) (2.10) we have 


$$
\begin{aligned}
\mathrm{d} X(t) \mathrm{d} \hat{y}(t) & =\pi^{\mathrm{T}} \Sigma \mathrm{d} v(t) \cdot\left(\Lambda \sigma^{\mathrm{T}}+\beta(t) A^{\mathrm{T}}\right)\left(\Sigma^{\mathrm{T}}\right)^{-1} \mathrm{~d} v(t) \\
& =\left(\Lambda \sigma^{\mathrm{T}}+\beta(t) A^{\mathrm{T}}\right)\left(\Sigma^{\mathrm{T}}\right)^{-1} \mathrm{~d} v(t) \mathrm{d} v(t)^{\mathrm{T}} \Sigma^{\mathrm{T}} \pi \\
& =\left(\Lambda \sigma^{\mathrm{T}}+\beta(t) A^{\mathrm{T}}\right) \pi \mathrm{d} t .
\end{aligned}
$$

By Itô's formula, it follows that

$$
\begin{aligned}
\mathcal{L} J= & J_{t}+J_{X}(r X+\bar{B} \pi)+J_{\hat{y}}^{\mathrm{T}}(d+D \hat{y})+\frac{1}{2} J_{X X} \pi^{\mathrm{T}} \Sigma \Sigma^{\mathrm{T}} \pi+J_{X \hat{y}}^{\mathrm{T}}\left(\Lambda \sigma^{\mathrm{T}}+\beta A^{\mathrm{T}}\right) \pi \\
& +\frac{1}{2} \operatorname{Tr}\left[\left(\Lambda \sigma^{\mathrm{T}}+\beta A^{\mathrm{T}}\right)\left(\Sigma \Sigma^{\mathrm{T}}\right)^{-1}\left(\Lambda \sigma^{\mathrm{T}}+\beta A^{\mathrm{T}}\right)^{\mathrm{T}} J_{\hat{y} \hat{y}}\right],
\end{aligned}
$$

where $J_{t}$ is the partial derivative of $J$ with respect to $t, J_{X X}$ is the second order partial derivative of $J$ with respect to $X$, and $J_{X}, J_{X \hat{y}}, J_{\hat{y} \hat{y}}$ are defined similarly. On the assumption that $J_{X X}>0$, we get the following optimal strategy

$$
\pi=-\left(\Sigma \Sigma^{\mathrm{T}}\right)^{-1}\left[\bar{B}^{\mathrm{T}} \frac{J_{X}}{J_{X X}}+\left(\Lambda \sigma^{\mathrm{T}}+\beta A^{\mathrm{T}}\right)^{\mathrm{T}} \frac{J_{X \hat{y}}}{J_{X X}}\right]
$$

which makes $\mathcal{L} J$ minimal. Substituting (3.2) into (3.15) leads to

$$
\left\{\begin{array}{l}
J_{t}+r X J_{X}+J_{\hat{y}}^{\mathrm{T}}(d+D \hat{y})+\frac{1}{2} \operatorname{Tr}\left[\left(\Lambda \sigma^{\mathrm{T}}+\beta A^{\mathrm{T}}\right)\left(\Sigma \Sigma^{\mathrm{T}}\right)^{-1}\left(\Lambda \sigma^{\mathrm{T}}+\beta A^{\mathrm{T}}\right)^{\mathrm{T}} J_{\hat{y} \hat{y}}\right] \\
\quad-\frac{1}{2}\left[\bar{B}^{\mathrm{T}} \frac{J_{X}}{J_{X X}}+\left(\Lambda \sigma^{\mathrm{T}}+\beta A^{\mathrm{T}}\right)^{\mathrm{T}} \frac{J_{X \hat{y}}}{J_{X X}}\right]^{\mathrm{T}}\left(\Sigma \Sigma^{\mathrm{T}}\right)^{-1}\left[\bar{B}^{\mathrm{T}} \frac{J_{X}}{J_{X X}}+\left(\Lambda \sigma^{\mathrm{T}}+\beta A^{\mathrm{T}}\right)^{\mathrm{T}} \frac{J_{X \hat{y}}}{J_{X X}}\right] J_{X X}=0, \\
J(T, X, \hat{y})=\frac{1}{2}(X-\beta)^{2} .
\end{array}\right.
$$

In this and the following PDEs and ODEs, the arguments $t, X, \hat{y}$ are always suppressed to simplify the notations.

Noticing that the terminal condition of $J$ is a nonhomogeneous function of $X$, in order to make (3.3) homogeneous, we set

$$
z(t)=X(t)-\alpha \mathrm{e}^{-\int_{t}^{T} r(s) \mathrm{d} s}
$$

Simple calculation shows

$$
\begin{aligned}
& J(t, X, \hat{y})=J\left(t, z+\alpha \mathrm{e}^{-\int_{t}^{T} r(s) \mathrm{d} s}, \hat{y}\right) \triangleq H(t, z, \hat{y}), \\
& H_{t}=J_{t}-\alpha J_{X} r(t) \mathrm{e}^{-\int_{t}^{T} r(s) \mathrm{d} s}, \\
& H_{X}=H_{z} z_{X}=H_{z}, \quad H_{X X}=H_{z z}, \quad H_{X \hat{y}}=H_{z \hat{y}} .
\end{aligned}
$$

Substituting $z$ and the above equalities into (3.3), we obtain that

$$
\left\{\begin{array}{l}
H_{t}+r z H_{z}+H_{\hat{y}}^{\mathrm{T}}(d+D \hat{y})+\frac{1}{2} \operatorname{Tr}\left[\left(\Lambda \sigma^{\mathrm{T}}+\beta A^{\mathrm{T}}\right)\left(\Sigma \Sigma^{\mathrm{T}}\right)^{-1}\left(\Lambda \sigma^{\mathrm{T}}+\beta A^{\mathrm{T}}\right)^{\mathrm{T}} H_{\hat{y} \hat{y}}\right] \\
\quad-\frac{1}{2}\left[\bar{B}^{\mathrm{T}} \frac{H_{z}}{H_{z z}}+\left(\Lambda \sigma^{\mathrm{T}}+\beta A^{\mathrm{T}}\right)^{\mathrm{T}} \frac{H_{z \hat{y}}}{H_{z z}}\right]^{\mathrm{T}}\left(\Sigma \Sigma^{\mathrm{T}}\right)^{-1}\left[\bar{B}^{\mathrm{T}} \frac{H_{z}}{H_{z z}}+\left(\Lambda \sigma^{\mathrm{T}}+\beta A^{\mathrm{T}}\right)^{\mathrm{T}} \frac{H_{z \hat{y}}}{H_{z z}}\right] H_{z z}=0, \\
H(T, z, \hat{y})=\frac{1}{2} z^{2} .
\end{array}\right.
$$

By the special structure of (3.5), the following separation form of $H(t, z, \hat{y})$ is taken

$$
H(t, z, \hat{y})=\frac{1}{2} f(t, \hat{y}) z^{2} \text {, with } f(T, \hat{y})=1 \text { for all } \hat{y}
$$


which will be proved in Theorem 3.1. Therefore, the optimal control (3.2) has the following structure

$$
\pi=-\left(\Sigma \Sigma^{\mathrm{T}}\right)^{-1}\left[\bar{B}^{\mathrm{T}}+\left(\Lambda \sigma^{\mathrm{T}}+\beta A^{\mathrm{T}}\right)^{\mathrm{T}} \frac{\partial \ln f}{\partial \hat{y}}\right] z
$$

which is linear in $z$, and (3.5) is equivalent to

$$
\left\{\begin{array}{l}
\frac{1}{2} \frac{\partial f}{\partial t} z^{2}+r f z^{2}+\frac{1}{2} \frac{\partial f^{\mathrm{T}}}{\partial \hat{y}}(d+D \hat{y}) z^{2}+\frac{1}{2} \operatorname{Tr}\left[\left(\Lambda \sigma^{\mathrm{T}}+\beta A^{\mathrm{T}}\right)\left(\Sigma \Sigma^{\mathrm{T}}\right)^{-1}\left(\Lambda \sigma^{\mathrm{T}}+\beta A^{\mathrm{T}}\right)^{\mathrm{T}} \frac{\partial^{2} f}{\partial \hat{y}^{2}}\right] z^{2} \\
\quad-\frac{1}{2}\left[\bar{B}^{\mathrm{T}} z+\left(\Lambda \sigma^{\mathrm{T}}+\beta A^{\mathrm{T}}\right)^{\mathrm{T}} \frac{\partial \ln f}{\partial \hat{y}} z\right]^{\mathrm{T}}\left(\Sigma \Sigma^{\mathrm{T}}\right)^{-1}\left[\bar{B}^{\mathrm{T}} z+\left(\Lambda \sigma^{\mathrm{T}}+\beta A^{\mathrm{T}}\right)^{\mathrm{T}} \frac{\partial \ln f}{\partial \hat{y}} z\right] f=0, \\
\frac{1}{2} f(T, \hat{y}) z^{2}=\frac{1}{2} z^{2} .
\end{array}\right.
$$

Clearly, if $f(t, \hat{y})$ solves the following PDE

$$
\left\{\begin{array}{l}
\frac{\partial f}{\partial t}+2 r f+\frac{\partial f^{\mathrm{T}}}{\partial \hat{y}}(d+D \hat{y})+\operatorname{Tr}\left[\left(\Lambda \sigma^{\mathrm{T}}+\beta A^{\mathrm{T}}\right)\left(\Sigma \Sigma^{\mathrm{T}}\right)^{-1}\left(\Lambda \sigma^{\mathrm{T}}+\beta A^{\mathrm{T}}\right)^{\mathrm{T}} \frac{\partial^{2} f}{\partial \hat{y}^{2}}\right] \\
\quad-\left[\bar{B}^{\mathrm{T}}+\left(\Lambda \sigma^{\mathrm{T}}+\beta A^{\mathrm{T}}\right)^{\mathrm{T}} \frac{\partial \ln f}{\partial \hat{y}}\right]^{\mathrm{T}}\left(\Sigma \Sigma^{\mathrm{T}}\right)^{-1}\left[\bar{B}^{\mathrm{T}}+\left(\Lambda \sigma^{\mathrm{T}}+\beta A^{\mathrm{T}}\right)^{\mathrm{T}} \frac{\partial \ln f}{\partial \hat{y}}\right] f=0, \\
f(T, \hat{y})=1, \\
\bar{B}(t)=(a+A \hat{y}(t))^{\mathrm{T}}-r(t) \mathbf{1},
\end{array}\right.
$$

then $H(t, z, \hat{y})$ has the explicit form of (3.6).

\subsection{Optimal Policy}

Notice that the left hand side of the first equation in (3.8) is linear in $f, \frac{\partial f}{\partial t}, \hat{y}, \frac{\partial^{2} f}{\partial \hat{y}^{2}}$, and quadratic in $\frac{\partial \ln f}{\partial \hat{y}}$. Therefore, we assume that $f$ has the following expression

$$
f(t, \hat{y})=\exp \left\{p(t)+q^{\mathrm{T}}(t) \hat{y}+\hat{y}^{\mathrm{T}} G(t) \hat{y}\right\}
$$

with $p(t) \in \mathbb{R}, q(t) \in \mathbb{R}^{n}, G(t) \in S^{n \times n}$ to be specified later. Here, $S^{n \times n}$ denotes the set of all symmetric $n \times n$ real matrices. The form (3.9) of $f$ enables us to get an equivalent equation that is independent of $f$ and is only a quadratic function of $\hat{y}$. Fixing the coefficients of the obtained equation to be zero, we can determine $p, q, G$ by solving several equations. Thus, we may prove that $H$ given in (3.6) satisfied the HJB Equation (3.1), indeed. Therefore, we have the following theorem.

Theorem 3.1. For problem (2.14), the optimal strategy is given by

$$
\begin{aligned}
\pi(t)=- & \left(\Sigma(t) \Sigma^{T}(t)\right)^{-1}\left[a^{\mathrm{T}}-r(t) \mathbf{1}+\left(\Lambda \sigma^{\mathrm{T}}(t)+\beta(t) A^{\mathrm{T}}\right)^{\mathrm{T}} q(t)\right. \\
& \left.+\left(A+\left(\Lambda \sigma^{\mathrm{T}}(t)+\beta(t) A^{\mathrm{T}}\right)^{\mathrm{T}} G(t)\right) \hat{y}(t)\right]\left(X(t)-\alpha \mathrm{e}^{-\int_{t}^{T} r(s) \mathrm{d} s}\right),
\end{aligned}
$$

where $\beta(t), q(t), G(t)$ are the unique solutions to the second equation of (2.8) and following ODEs, respectively,

$$
\left\{\begin{array}{l}
\frac{\mathrm{d} q^{\mathrm{T}}}{\mathrm{d} t}+\left[q^{\mathrm{T}} D+d^{\mathrm{T}} G\right]-2\left(a^{\mathrm{T}}-r \mathbf{1}\right)\left(\Sigma \Sigma^{\mathrm{T}}\right)^{-1} A-2\left(a^{\mathrm{T}}-r \mathbf{1}\right)\left(\Sigma \Sigma^{\mathrm{T}}\right)^{-1}\left(\Lambda \sigma^{\mathrm{T}}+\beta A^{\mathrm{T}}\right)^{\mathrm{T}} G \\
\quad-2 q^{\mathrm{T}}\left(\Lambda \sigma^{\mathrm{T}}+\beta A^{\mathrm{T}}\right)\left(\Sigma \Sigma^{\mathrm{T}}\right)^{-1} A=0, \\
q(T)=0,
\end{array}\right.
$$




$$
\left\{\begin{array}{l}
\frac{\mathrm{d} G}{\mathrm{~d} t}-A^{\mathrm{T}}\left(\Sigma \Sigma^{\mathrm{T}}\right)^{-1} A-\left[A^{\mathrm{T}}\left(\Sigma \Sigma^{\mathrm{T}}\right)^{-1}\left(\Lambda \sigma^{\mathrm{T}}+\beta A^{\mathrm{T}}\right)^{\mathrm{T}}+D^{\mathrm{T}}\right] G-G\left[\left(\Lambda \sigma^{\mathrm{T}}+\beta A^{\mathrm{T}}\right)\left(\Sigma \Sigma^{\mathrm{T}}\right)^{-1} A+D\right]=0, \\
G(T)=0 .
\end{array}\right.
$$

Proof. Bearing the form (3.9) of $f$ in mind, simple calculation shows that

$$
\begin{aligned}
& \frac{\partial f}{\partial \hat{y}}=f(q+G \hat{y}), \\
& \frac{\partial^{2} f}{\partial \hat{y}^{2}}=f\left(q q^{\mathrm{T}}+q \hat{y}^{\mathrm{T}} G+G \hat{y} q^{\mathrm{T}}+G \hat{y} \hat{y}^{\mathrm{T}} G+G\right), \\
& \frac{\partial \ln f}{\partial \hat{y}}=q+G \hat{y}, \\
& \frac{\partial f}{\partial t}=f\left(\frac{\mathrm{d} p(t)}{\mathrm{d} t}+\frac{\mathrm{d} q(t)}{\mathrm{d} t} \hat{y}+\hat{y}^{\mathrm{T}} \frac{\mathrm{d} G(t)}{\mathrm{d} t} \hat{y}\right) .
\end{aligned}
$$

Therefore, (3.8) is equivalent to

$$
\left\{\begin{aligned}
& \frac{\mathrm{d} p}{\mathrm{~d} t}+ \frac{\mathrm{d} q^{\mathrm{T}}}{\mathrm{d} t} \hat{y}+\hat{y}^{\mathrm{T}} \frac{\mathrm{d} G}{\mathrm{~d} t} \hat{y}+2 r+(q+G \hat{y})^{\mathrm{T}}(d+D \hat{y}) \\
&+\operatorname{Tr}\left[\left(\Lambda \sigma^{\mathrm{T}}+\beta A^{\mathrm{T}}\right)\left(\Sigma \Sigma^{\mathrm{T}}\right)^{-1}\left(\Lambda \sigma^{\mathrm{T}}+\beta A^{\mathrm{T}}\right)^{\mathrm{T}}\left(q q^{\mathrm{T}}+q \hat{y}^{\mathrm{T}} G+G \hat{y} q^{\mathrm{T}}+G \hat{y} \hat{y}^{\mathrm{T}} G+G\right)\right] \\
& \quad\left[\bar{B}^{\mathrm{T}}+\left(\Lambda \sigma^{\mathrm{T}}+\beta A^{\mathrm{T}}\right)^{\mathrm{T}}(q+G \hat{y})\right]^{\mathrm{T}}\left(\Sigma \Sigma^{\mathrm{T}}\right)^{-1} \times\left[\bar{B}^{\mathrm{T}}+\left(\Lambda \sigma^{\mathrm{T}}+\beta A^{\mathrm{T}}\right)^{\mathrm{T}}(q+G \hat{y})\right]=0, \\
& p(T)+q^{\mathrm{T}}(T) \hat{y}(T)+\hat{y}^{\mathrm{T}}(T) G(T) \hat{y}(T)=0,
\end{aligned}\right.
$$

which is equivalent to

$$
\left\{\begin{aligned}
& \frac{\mathrm{d} p}{\mathrm{~d} t}+ \frac{\mathrm{d} q^{\mathrm{T}}}{\mathrm{d} t} \hat{y}+\hat{y}^{\mathrm{T}} \frac{\mathrm{d} G}{\mathrm{~d} t} \hat{y}+2 r+(q+G \hat{y})^{\mathrm{T}}(d+D \hat{y})+\operatorname{Tr}\left[\left(\Lambda \sigma^{\mathrm{T}}+\beta A^{\mathrm{T}}\right)\left(\Sigma \Sigma^{\mathrm{T}}\right)^{-1}\left(\Lambda \sigma^{\mathrm{T}}+\beta A^{\mathrm{T}}\right)^{\mathrm{T}} G\right] \\
& \quad\left[(a+A \hat{y})^{\mathrm{T}}-r \mathbf{1}\right]\left(\Sigma \Sigma^{\mathrm{T}}\right)^{-1}\left[(a+A \hat{y})^{\mathrm{T}}-r \mathbf{1}\right]^{\mathrm{T}}-2\left[(a+A \hat{y})^{\mathrm{T}}-r \mathbf{1}\right]\left(\Sigma \Sigma^{\mathrm{T}}\right)^{-1}\left(\Lambda \sigma^{\mathrm{T}}+\beta A^{\mathrm{T}}\right)^{\mathrm{T}}(q+G \hat{y})=0, \\
& p(T)+q^{\mathrm{T}}(T) \hat{y}(T)+\hat{y}^{\mathrm{T}}(T) G(T) \hat{y}(T)=0 .
\end{aligned}\right.
$$

The left hand of above PDE can be decomposed into three terms:

1) the term that is irrespective of $\hat{y}$

$$
\begin{aligned}
& \frac{\mathrm{d} p}{\mathrm{~d} t}+q^{\mathrm{T}}\left(\Lambda \sigma^{\mathrm{T}}+\beta A^{\mathrm{T}}\right)\left(\Sigma \Sigma^{\mathrm{T}}\right)^{-1}\left(\Lambda \sigma^{\mathrm{T}}+\beta A^{\mathrm{T}}\right)^{\mathrm{T}} q-\left[2\left(a^{\mathrm{T}}-r \mathbf{1}\right)\left(\Sigma \Sigma^{\mathrm{T}}\right)^{-1}\left(\Lambda \sigma^{\mathrm{T}}+\beta A^{\mathrm{T}}\right)^{\mathrm{T}}-d^{\mathrm{T}}\right] q \\
& \quad+2 r-\left(a^{\mathrm{T}}-r \mathbf{1}\right)\left(\Sigma \Sigma^{\mathrm{T}}\right)^{-1}\left(a^{\mathrm{T}}-r \mathbf{1}\right)^{\mathrm{T}}+\operatorname{Tr}\left[\left(\Lambda \sigma^{\mathrm{T}}+\beta A^{\mathrm{T}}\right)\left(\Sigma \Sigma^{\mathrm{T}}\right)^{-1}\left(\Lambda \sigma^{\mathrm{T}}+\beta A^{\mathrm{T}}\right)^{\mathrm{T}} G\right]
\end{aligned}
$$

2) the term that is linear in $\hat{y}$

$$
\begin{aligned}
& \frac{\mathrm{d} q^{\mathrm{T}}}{\mathrm{d} t} \hat{y}+\left[q^{\mathrm{T}} D+d^{\mathrm{T}} G\right] \hat{y}-2\left(a^{\mathrm{T}}-r \mathbf{1}\right)\left(\Sigma \Sigma^{\mathrm{T}}\right)^{-1} A \hat{y}-2\left(a^{\mathrm{T}}-r \mathbf{1}\right)\left(\Sigma^{2}\right)^{-1}\left(\Lambda \sigma^{\mathrm{T}}+\beta A^{\mathrm{T}}\right)^{\mathrm{T}} G \hat{y} \\
& \quad-2 q^{\mathrm{T}}\left(\Lambda \sigma^{\mathrm{T}}+\beta(t) A^{\mathrm{T}}\right)\left(\Sigma \Sigma^{\mathrm{T}}\right)^{-1} A \hat{y}
\end{aligned}
$$

3) the term that is quadratic in $\hat{y}$

$$
\hat{y}^{\mathrm{T}}\left(\frac{\mathrm{d} G}{\mathrm{~d} t}+G D-A^{\mathrm{T}}\left(\Sigma \Sigma^{\mathrm{T}}\right)^{-1} A-2 A^{\mathrm{T}}\left(\Sigma \Sigma^{\mathrm{T}}\right)^{-1}\left(\Lambda \sigma^{\mathrm{T}}+\beta A^{\mathrm{T}}\right)^{\mathrm{T}} G\right) \hat{y} .
$$


So, if the $p, q, G$ satisfy the following three equations, respectively,

$$
\begin{aligned}
& \left\{\begin{array}{l}
\frac{\mathrm{d} p}{\mathrm{~d} t}+q^{\mathrm{T}}\left(\Lambda \sigma^{\mathrm{T}}+\beta A^{\mathrm{T}}\right)\left(\Sigma \Sigma^{\mathrm{T}}\right)^{-1}\left(\Lambda \sigma^{\mathrm{T}}+\beta A^{\mathrm{T}}\right)^{\mathrm{T}} q+2 r-\left[2\left(a^{\mathrm{T}}-r \mathbf{1}\right)\left(\Sigma^{2}\right)^{-1}\left(\Lambda \sigma^{\mathrm{T}}+\beta A^{\mathrm{T}}\right)^{\mathrm{T}}-d^{\mathrm{T}}\right] q \\
\quad-\left(a^{\mathrm{T}}-r \mathbf{1}\right)\left(\Sigma \Sigma^{\mathrm{T}}\right)^{-1}\left(a^{\mathrm{T}}-r \mathbf{1}\right)^{\mathrm{T}}+\operatorname{Tr}\left[\left(\Lambda \sigma^{\mathrm{T}}+\beta A^{\mathrm{T}}\right)\left(\Sigma \Sigma^{\mathrm{T}}\right)^{-1}\left(\Lambda \sigma^{\mathrm{T}}+\beta A^{\mathrm{T}}\right)^{\mathrm{T}} G\right]=0, \\
p(T)=0,
\end{array}\right. \\
& \qquad \begin{array}{r}
\frac{\mathrm{d} q^{\mathrm{T}}}{\mathrm{d} t}+\left[q^{\mathrm{T}} D+d^{\mathrm{T}} G\right]-2\left(a^{\mathrm{T}}-r \mathbf{1}\right)\left(\Sigma \Sigma^{\mathrm{T}}\right)^{-1} A-2\left(a^{\mathrm{T}}-r \mathbf{1}\right)\left(\Sigma \Sigma^{\mathrm{T}}\right)^{-1}\left(\Lambda \sigma^{\mathrm{T}}+\beta A^{\mathrm{T}}\right)^{\mathrm{T}} G \\
\quad-2 q^{\mathrm{T}}\left(\Lambda \sigma^{\mathrm{T}}+\beta A^{\mathrm{T}}\right)\left(\Sigma \Sigma^{\mathrm{T}}\right)^{-1} A=0, \\
q(T)=0,
\end{array} \\
& \left\{\begin{array}{l}
\frac{\mathrm{d} G}{\mathrm{~d} t}-A^{\mathrm{T}}\left(\Sigma \Sigma^{\mathrm{T}}\right)^{-1} A-\left[A^{\mathrm{T}}\left(\Sigma \Sigma^{\mathrm{T}}\right)^{-1}\left(\Lambda \sigma^{\mathrm{T}}+\beta A^{\mathrm{T}}\right)^{\mathrm{T}}-\frac{1}{2} D^{\mathrm{T}}\right] G-G\left[\left(\Lambda \sigma^{\mathrm{T}}+\beta A^{\mathrm{T}}\right)\left(\Sigma \Sigma^{\mathrm{T}}\right)^{-1} A-\frac{1}{2} D\right]=0, \\
G(T)=0,
\end{array}\right.
\end{aligned}
$$

we can determine the function $f$. Firstly, we need to claim that the second equation of (2.8), (3.15), (3.16) and (33.17) have unique solution. In fact, it is known that the second equation of (2.8) has a unique nonnegative definite solution; see, for example, Theorem 10.3 of [20]. While for (3.17), (3.16) and (3.15), they are linear in $G, q, p$, respectively; thus, the solutions exist uniquely. This means that $f$ given in (3.9) exactly solves (3.8). Furthermore, by the analysis in the above subsection, we can conclude that $H$ defined in (3.6) solves (3.3). Notice that

$$
J_{X X}(t)=H_{z z}(t)=f(t, \hat{y})=\exp \left\{p(t)+q(t)^{\mathrm{T}} \hat{y}+\hat{y}^{\mathrm{T}} G(t) \hat{y}\right\}>0 .
$$

Thus, $H$ defined in (3.6) satisfies HJB Equation (3.1). Clearly, (3.2) is equal to (3.10). In the end, we need only to confirm that (3.10) is admissible. By classic filtering theory, $\mathcal{G}_{t}$ is equal to the $\sigma$-algebra generated by innovation process $\left\{v_{s}, s \leq t\right\}$ (see for example [21]). Clearly, we have (3.10) is $\sigma\left(v_{s}, s \leq t\right)$-adapted, and thus it is admissible. Therefore, (3.10) is the optimal strategy, which make the $\frac{1}{2} E[X(T)-\alpha]^{2}$ minimal. This completes the proof.

We will give a brief discussion about the solvability in theory of (2.8) (3.11) (3.12). Clearly, $\beta(t)$ satisfies

$$
\dot{\beta}=\left(D-\Lambda \sigma^{\mathrm{T}} \Gamma^{-1} A\right) \beta+\beta(t)\left(D^{\mathrm{T}}-A^{\mathrm{T}} \Gamma^{-1} \sigma \Lambda^{\mathrm{T}}\right)-\beta A^{\mathrm{T}} \Gamma^{-1} A \beta+\Lambda \Lambda^{\mathrm{T}}-\Lambda \sigma^{\mathrm{T}} \Gamma^{-1} \sigma \Lambda^{\mathrm{T}}
$$

with $\beta(0)=0$. Let $\bar{\beta}(s)=\beta(t), s=T-t$, then it follows

$$
-\frac{\mathrm{d} \bar{\beta}}{\mathrm{d} s}=\left(D-\Lambda \sigma^{\mathrm{T}} \Gamma^{-1} A\right) \bar{\beta}+\bar{\beta}\left(D^{\mathrm{T}}-A^{\mathrm{T}} \Gamma^{-1} \sigma \Lambda^{\mathrm{T}}\right)-\bar{\beta} A^{\mathrm{T}} \Gamma^{-1} A \bar{\beta}+\Lambda \Lambda^{\mathrm{T}}-\Lambda \sigma^{\mathrm{T}} \Gamma^{-1} \sigma \Lambda^{\mathrm{T}}
$$

with $\bar{\beta}(T)=0$. By known result (see for example Anderson and Moore (1971)), $\bar{\beta}(s)$ can be represented as

$$
\bar{\beta}(s)=K(s) L^{-1}(s)
$$

where $K(s), L(s)$ are defined as

$$
\left[\begin{array}{c}
\dot{K} \\
\dot{L}
\end{array}\right]=\left[\begin{array}{cc}
D^{\mathrm{T}}-A^{\mathrm{T}} \Gamma^{-1} \sigma \Lambda^{\mathrm{T}} & -A^{\mathrm{T}} \Gamma^{-1} A \\
-\Lambda \Lambda^{\mathrm{T}}+\Lambda \sigma^{\mathrm{T}} \Gamma^{-1} \sigma \Lambda^{\mathrm{T}} & -D+\Lambda \sigma^{\mathrm{T}} \Gamma^{-1} A
\end{array}\right]\left[\begin{array}{c}
K \\
L
\end{array}\right], \quad\left[\begin{array}{c}
K(T) \\
L(T)
\end{array}\right]=\left[\begin{array}{l}
I \\
0
\end{array}\right] .
$$

Therefore,

$$
\beta(t)=\bar{\beta}(s)=K(T-t) L^{-1}(T-t) .
$$


Clearly, (3.12) is a Lyapunov differential equation, which is solved by introducing the following operator

$$
\operatorname{Vec}(G(t))=\left(G(t)^{(1) \mathrm{T}}, G(t)^{(2) \mathrm{T}}, \cdots, G(t)^{(n) \mathrm{T}}\right)^{T} \triangleq \mathbb{G}(t) \in \mathbb{R}^{n^{2}}
$$

where $G(t)^{(i) \mathrm{T}}$ is the transpose of $i$-th column of of $G$. Clearly,

$$
\dot{\mathbb{G}}(t)=\mathbb{P}^{G_{1}}(t) \mathbb{G}(t)+\mathbb{P}^{G_{2}}(t), \quad \mathbb{G}(T)=0
$$

where

$$
\begin{aligned}
& \mathbb{P}^{G_{1}}(t)=I \otimes\left[\left(\Lambda \sigma^{\mathrm{T}}+\beta(t) A^{\mathrm{T}}\right)\left(\Sigma(t) \Sigma^{\mathrm{T}}(t)\right)^{-1} A-\frac{1}{2} D\right]^{\mathrm{T}}+\left[\left(\Lambda \sigma^{\mathrm{T}}(t)+\beta(t) A^{\mathrm{T}}\right)\left(\Sigma(t) \Sigma^{\mathrm{T}}(t)\right)^{-1} A-\frac{1}{2} D\right] \otimes I, \\
& \mathbb{P}^{G_{2}}(t)=\operatorname{Vec}\left(A^{\mathrm{T}}\left(\Sigma^{2}\right)^{-1} A\right) .
\end{aligned}
$$

Let $\overline{\mathbb{G}}(s)=\mathbb{G}_{T-s}$. Then

$$
\frac{\mathrm{d} \overline{\mathbb{G}}}{\mathrm{d} s}=-\mathbb{P}^{G_{1}}(T-s) \overline{\mathbb{G}}(s)-\mathbb{P}^{G_{2}}(T-s), \quad \overline{\mathbb{G}}_{0}=0 .
$$

Therefore,

$$
\mathbb{G}(t)=\overline{\mathbb{G}}(s)=-\int_{0}^{s} \Phi(s, \tau) \mathbb{P}^{G_{2}}(T-\tau) \mathrm{d} \tau
$$

where $\Phi(\cdot, \cdot)$ is the fundamental matrix of (3.18). Thus $G(t)=\operatorname{Vec}^{-1}(\mathbb{G}(t))$. At last, (3.11) and (3.15) can be easily solved by the linearity of the equations.

\section{Efficient Frontier}

In this section, we proceed to derive the efficient frontier for the original portfolio selection problem under partial information. To begin with, we prove a lemma which shows the feasibility of the original problem.

Lemma 4.1. Problem (5) is feasible, and the minimal mean-variance of the terminal wealth process is finite.

Proof. The proof follows directly from results of Section 5 in [17]. In the language of [17], (2.10) can be rewritten as

$$
\left\{\begin{array}{l}
\mathrm{d} X(t)=\left[r(t) X(t)+\bar{B}(t) \Sigma^{T} Z(t)\right] \mathrm{d} t+Z(t) \mathrm{d} v_{t}, \\
X(T)=v
\end{array}\right.
$$

where $v$ is defined by Theorem 5.4 in [17] satisfying $E v=\bar{x}$, and

$$
Z(t)=\left(\Sigma^{\mathrm{T}}(t)\right)^{-1} \pi(t) \text {. }
$$

Clearly, $\mathcal{G}_{t}$ is equivalent to the $\sigma$-algebra generated by innovation process $\left\{v_{u}, u \leq t\right\}$. By general BSDEs theory, (4.1) has a unique $\mathcal{G}_{t}$-adapted, square integrate solution $(X(\cdot), Z(\cdot))$. Therefore, problem (2.5) is feasible because $\Sigma^{\mathrm{T}}(x) Z(t)$ is a feasible strategy. On the other hand, by Theorem 5.6 of [17], we know that the minimal mean-variance at the terminal time point is finite.

Now, we state our main theorem.

Theorem 4.1. The efficient strategy of Problem (2.5) with the terminal expected wealth constraint $E X(T)=\bar{X}$ is given by

$$
\begin{aligned}
\pi(t)= & -\left(\Sigma(t) \Sigma^{\mathrm{T}}(t)\right)^{-1}\left[a^{\mathrm{T}}-r(t) \mathbf{1}+\left(\Lambda \sigma^{\mathrm{T}}(t)+\beta(t) A^{\mathrm{T}}\right)^{\mathrm{T}} q(t)\right. \\
& \left.+\left(A+\left(\Lambda \sigma^{\mathrm{T}}(t)+\beta(t) A^{\mathrm{T}}\right)^{\mathrm{T}} G(t)\right) \hat{y}(t)\right]\left(X(t)-\left(\bar{x}+\gamma^{*}\right) \mathrm{e}^{-\int_{t}^{\mathrm{T}} r(s) \mathrm{d} s}\right) .
\end{aligned}
$$

Here, $\beta(t), q(t), G(t)$ solve Equations (2.8) (3.11) (3.12), respectively, and $\gamma^{*}$ is given by

$$
\gamma^{*}=\frac{\bar{x}-x_{0} \mathrm{e}^{\int_{t}^{T} r(s) \mathrm{d} s}}{\mathrm{e}^{2 \int_{t}^{T} r(s) \mathrm{d} s} E\left[\mathrm{e}^{2 \xi_{T}}\right]-1} E\left[\mathrm{e}^{2 \xi_{T}}\right]
$$


where $\xi_{T}$ is given by

$$
\begin{aligned}
\xi_{T}=\int_{0}^{T} & {\left[r(s)-\bar{B}(s)\left(\Sigma \Sigma^{\mathrm{T}}\right)^{-1}(V(s)+U(s) \hat{y}(s))\right] \mathrm{d} s+\int_{0}^{T}[V(s)+U(s) \hat{y}(s)]^{\mathrm{T}} \Sigma^{-1} \mathrm{~d} v(s) } \\
& -\frac{1}{2} \int_{0}^{T}\left|[V(s)+U(s) \hat{y}(s)]^{\mathrm{T}} \Sigma^{-1}\right|^{2} \mathrm{~d} s,
\end{aligned}
$$

and $V(t)=a^{\mathrm{T}}-r(t) \mathbf{1}+\left(\Lambda \sigma^{\mathrm{T}}(t)+\beta(t) A^{\mathrm{T}}\right)^{\mathrm{T}} q(t), \quad U(t)=A+\left(\Lambda \sigma^{\mathrm{T}}(t)+\beta(t) A^{\mathrm{T}}\right)^{\mathrm{T}} G(t)$. Moreover, the efficient frontier is given by

$$
\frac{1}{2}\left[x_{0}-\left(\bar{x}+\gamma^{*}\right) \mathrm{e}^{-\int_{t}^{T} r(s) \mathrm{ds}}\right]^{2} E\left[\mathrm{e}^{2 \xi_{T}}\right]-\frac{1}{2} \gamma^{* 2} .
$$

Proof. By Lemma 4.1, we know that the constraint Problem (2.5) is feasible, and its minimal terminal meanvariance $J^{*}$ is finite. This means that

$$
J^{*}=\max _{\gamma \in R} \min _{\pi \in \Pi}\left\{\frac{1}{2} E[X(T)-\bar{x}]^{2}-\gamma[E X(T)-\bar{x}]\right\}<\infty
$$

where the equality is true by general convex constraint optimization theory (see, for example, [22]). By Theorem 3.1, the wealth Equation (2.10) evolves as

$$
\begin{aligned}
\mathrm{d} X(t)= & \left\{r(t) X(t)-\bar{B}(t)\left(\Sigma(t) \Sigma^{\mathrm{T}}(t)\right)^{-1}[V(t)+U(t) \hat{y}(t)]\left(X(t)-\alpha \mathrm{e}^{-\int_{t}^{T} r(s) \mathrm{ds}}\right)\right\} \mathrm{d} t \\
& -\left(X(t)-\alpha \mathrm{e}^{-\int_{t}^{T} r(s) \mathrm{d} s}\right)[V(t)+U(t) \hat{y}(t)]^{\mathrm{T}}\left(\Sigma(t) \Sigma^{\mathrm{T}}(t)\right)^{-1} \Sigma(t) \mathrm{d} v(t) .
\end{aligned}
$$

In terms of $z$, this equation is

$$
\left\{\begin{array}{l}
\mathrm{d} z(t)=\left\{r(t)-\bar{B}(t)\left(\Sigma \Sigma^{\mathrm{T}}\right)^{-1}[V(t)+U(t) \hat{y}(t)]\right\} z(t) \mathrm{d} t-z(t)[V(t)+U(t) \hat{y}(t)]^{\mathrm{T}} \Sigma^{-1} \mathrm{~d} v(t), \\
z(0)=x_{0}-\alpha \mathrm{e}^{-\int_{t}^{T} r(s) \mathrm{d} s} \triangleq z_{0} .
\end{array}\right.
$$

Clearly,

$$
\begin{aligned}
z(t)= & z_{0} \exp \left\{\int_{0}^{t}\left[r(s)-\bar{B}(s)\left(\Sigma \Sigma^{\mathrm{T}}\right)^{-1}(V(s)+U(s) \hat{y}(s))\right] \mathrm{d} t\right. \\
& \left.+\int_{0}^{t}[V(s)+U(s) \hat{y}(s)]^{\mathrm{T}} \Sigma^{-1} \mathrm{~d} v(s)-\frac{1}{2} \int_{0}^{t}\left|[V(s)+U(s) \hat{y}(s)]^{\mathrm{T}} \Sigma^{-1}\right|^{2} \mathrm{~d} s\right\} .
\end{aligned}
$$

Thus

$$
E[z(T)]^{2}=z_{0}^{2} E\left[\mathrm{e}^{2 \xi_{T}}\right]
$$

where $\xi_{T}$ is defined in (4.3). Notice that

$$
\frac{1}{2} E[z(T)]^{2}=\frac{1}{2} E[X(T)-\bar{x}]^{2}-\gamma[E X(T)-\bar{x}]+\frac{1}{2} \gamma^{2} .
$$

For any fixed $\gamma$,

$$
\min _{\pi \in \Pi}\left\{\frac{1}{2} E[X(T)-\bar{x}]^{2}-\gamma[E X(T)-\bar{x}]\right\}=\frac{1}{2}\left[x_{0}-(\bar{x}+\gamma) \mathrm{e}^{-\int_{t}^{T} r(s) \mathrm{ds}}\right]^{2} E\left[\mathrm{e}^{2 \xi_{T}}\right]-\frac{1}{2} \gamma^{2} \triangleq \mathbb{J}(\gamma) .
$$

To obtain the optimal mean-variance value and the optimal portfolio strategy of Problem (2.5), we should maximize (4.6) over $\gamma$ within $R$, and the finiteness is ensured by (4.5). We easily show that (4.6) attains its maximum value $\mathbb{J}\left(\gamma^{*}\right)$ at

$$
\gamma^{*}=\frac{\bar{X}-x_{0} \mathrm{e}^{\int_{t}^{T} r(s) \mathrm{d} s}}{\mathrm{e}^{2 \int_{t}^{T} r(s) \mathrm{d} s} E\left[\mathrm{e}^{2 \xi_{T}}\right]-1} E\left[\mathrm{e}^{2 \xi_{T}}\right]
$$


And we can assert that

$$
\mathrm{e}^{2 \int_{t}^{T} r(s) \mathrm{d} s} E\left[\mathrm{e}^{2 \xi_{T}}\right]-1 \neq 0
$$

If this is not true, the optimal cost will be infinite, which contradicts (4.5).

\section{Conclusion}

In this paper, we have studied the continuous-time mean-variance portfolio selection problem with stochastic drifts. In particular, drifts are assumed to be linear functions of economic factor processes. Because the factor processes cannot be observed directly, partial information is assumed together with a filter process. Consequently, by dynamic programming technique and the method of separation of variables, we have derived the explicit optimal strategy via the solution of a system of ODEs. As a future extension, it would be of interest to study the solutions with real financial data and carry out appropriate economic analysis. Also, regime-switching model [23] and the scenario for no-bankruptcy can also be considered.

\section{Acknowledgements}

This work is supported by the PolyU grant G-YL05 and A-PL62, and the JRI of the Department of Applied Mathematics, The Hong Kong Polytechnic University.

\section{References}

[1] Markowitz, H. (1952) Portfolio Selection. Journal of Finance, 7, 77-91.

[2] Markowitz, H. (1959) Portfolio Selection: Efficient Diversification of Investment. John Wiley \& Sons, New York.

[3] Sharpe, W.F. (1964) Capital Asset Prices: A Theory of Market Equilibrium under Conditions of Risk. Journal of Finance, 19, 425-442.

[4] Merton, R. (1971) Optimum Consumption and Portfolio Rules in a Continuous Time Model. Journal of Economic Theory, 3, 373-413. http://dx.doi.org/10.1016/0022-0531(71)90038-X

[5] Pliska, S.R. (1986) A Stochastic Calculus Model of Continuous Trading: Optimal Portfolios. Mathematics of Operations Research, 11, 371-384. http://dx.doi.org/10.1287/moor.11.2.371

[6] Zhou, X.Y. and Li, D. (2000) Continuous-Time Mean-Variance Portfolio Selection: A Stochastic LQ Framework. Applied Mathematics and Optimization, 42, 19-33. http://dx.doi.org/10.1007/s002450010003

[7] Lim, A.E.B. and Zhou, X.Y. (2002) Mean-Variance Portfolio Selection with Random Parameters in a Complete Market. Mathematics of Operations Research, 27, 101-120. http://dx.doi.org/10.1287/moor.27.1.101.337

[8] Zhou, X.Y. and Yin, G. (2003) Markowitz’s Mean-Variance Portfolio Selection with Regime Switching: A Continuous Time Model. SIAM Journal on Control and Optimization, 42, 1466-1482. http://dx.doi.org/10.1137/S0363012902405583

[9] Li, D. and Ng, W.L. (2000) Optimal Dynamic Portfolio Selection: Multi-Period Mean-Variance Formulation. Mathematical Finance, 10, 387-406. http://dx.doi.org/10.1111/1467-9965.00100

[10] Steinbach, M.C. (2001) Markowitz Revisited: Mean-Variance Models in Financial Portfolio Analysis. SIAM Review, 43, 31-85. http://dx.doi.org/10.1137/S0036144500376650

[11] Bielecki, T.R., Jin, H., Pliska, S.R. and Zhou, X.Y. (2005) Continuous-Time Mean-Variance Portfolio Selection with Bankruptcy Prohibition. Mathematical Finance, 15, 213-244. http://dx.doi.org/10.1111/j.0960-1627.2005.00218.x

[12] Bielecki, T.R. and Pliska, S.R. (1999) Risk-Sensitive Dynamic Asset Management. Applied Mathematics and Optimization, 39, 337-360. http://dx.doi.org/10.1007/s002459900110

[13] Nagai, H. and Peng, S.G. (2002) Risk-Sensitive Dynamic Portfolio Optimization with Partial Information on Infinite Time Horizon. The Annals of Applied Probability, 12, 173-195. http://dx.doi.org/10.1214/aoap/1015961160

[14] Bielecki, T.R. and Pliska, S.R. (2004) Risk-Sensitive ICAPM with Application to Fixed-Income Management. IEEE Transactions on Automatic Control, 49, 420-432. http://dx.doi.org/10.1109/TAC.2004.824470

[15] Lakner, P. (1995) Utility Maximization with Partial Information. Stochastic Processes and Their Applications, 56, 247273. http://dx.doi.org/10.1016/0304-4149(94)00073-3

[16] Lakner, P. (1998) Optimal Trading Strategy for an Investor: The Case of Partial Information. Stochastic Processes and Their Applications, 76, 77-97. http://dx.doi.org/10.1016/S0304-4149(98)00032-5

[17] Xiong, J. and Zhou, X.Y. (2007) Mean-Variance Portfolio Selection under Partial Information. SIAM Journal on Con- 
trol and Optimization, 46, 156-175. http://dx.doi.org/10.1137/050641132

[18] Karatzas, I. and Shreve, S.E. (1991) Methods of Mathematical Finance. Springer-Verlag, New York.

[19] Li, X. and Zhou, X.Y. (2006) Continuous-Time Mean-Variance Efficiency: The 80\% Rule. Annals of Applied Probability, 16, 1751-1763. http://dx.doi.org/10.1214/105051606000000349

[20] Liptser, R.S. and Shiryaev, A.N. (2001) Statistics of Random Processes: I. General Theory. Springer, Berlin.

[21] Kallianpur, G. (1980) Stochastic Filtering Theory. Springer-Verlag, New York. http://dx.doi.org/10.1007/978-1-4757-6592-2

[22] Luenberger, D.G. (1968) Optimization by Vector Space Method. John Wiley, New York.

[23] Yiu, K.F.C., Liu, J.Z., Siu, T.K. and Ching, W.K. (2010) Optimal Portfolios with Regime-Switching and Value-at-Risk Constraint. Automatica, 46, 979-989. http://dx.doi.org/10.1016/j.automatica.2010.02.027 
Scientific Research Publishing (SCIRP) is one of the largest Open Access journal publishers. It is currently publishing more than 200 open access, online, peer-reviewed journals covering a wide range of academic disciplines. SCIRP serves the worldwide academic communities and contributes to the progress and application of science with its publication.

Other selected journals from SCIRP are listed as below. Submit your manuscript to us via either submit@scirp.org or Online Submission Portal.
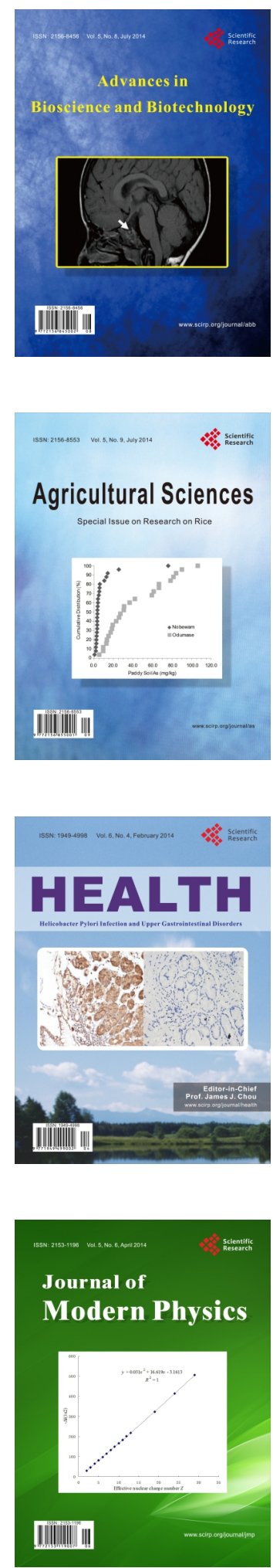
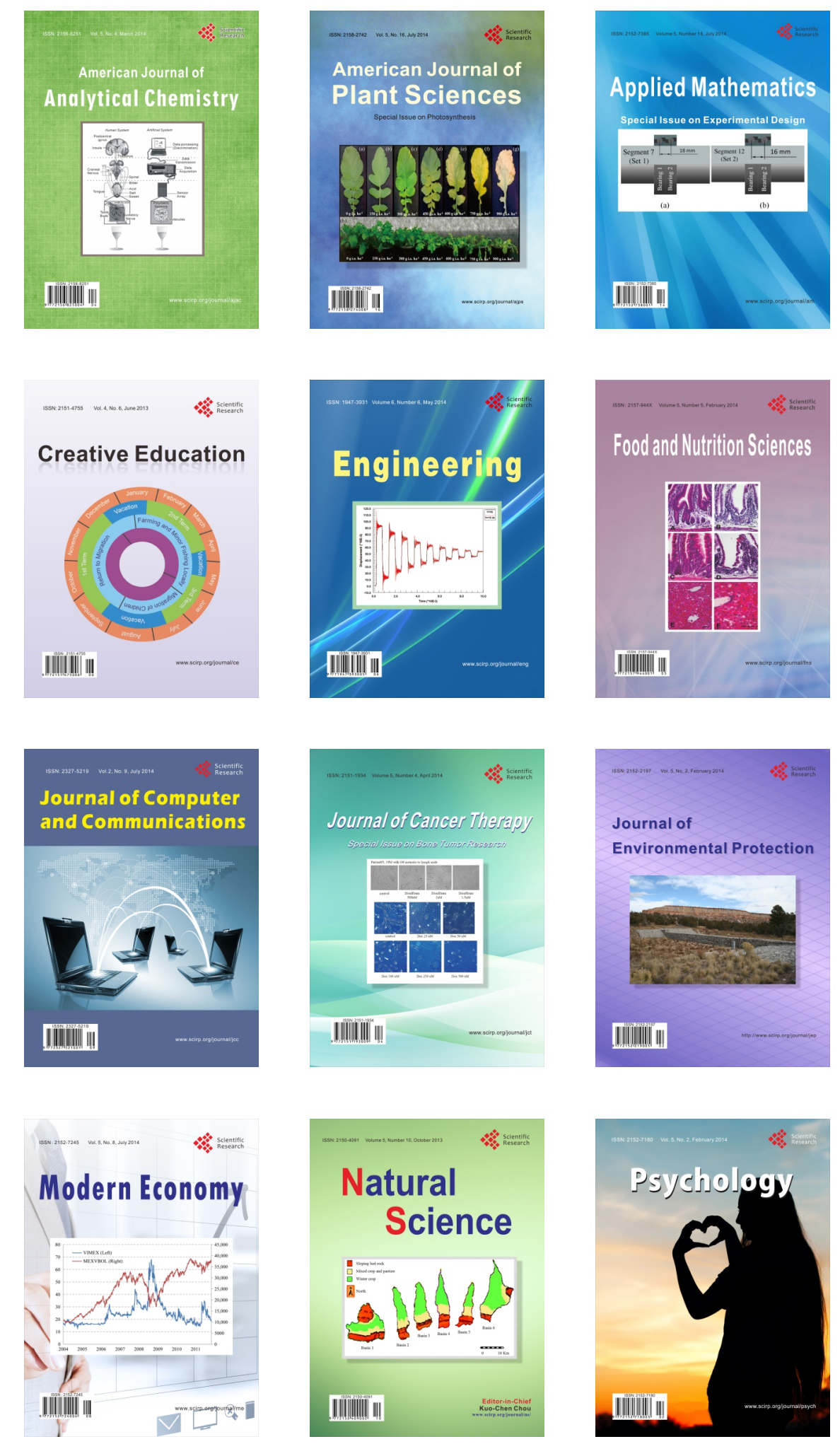